\title{
Computing the long-distance contributions to $\varepsilon_{K}$
}

\section{Ziyuan Bai}

Columbia University, USA

bzyhtydgmail.com

Norman Christ* ${ }^{\dagger}$

Columbia University, USA

E-mail: nhcephys.columbia.edu

\section{RBC and UKQCD Collaborations}

We will discuss the theoretical framework and initial results for the lattice QCD calculation of the long-distance contributions to $\varepsilon_{K}$, the measure of indirect $\mathrm{CP}$ violation in $K_{L}$ decay. A proofof-principle calculation has been carried out on a $24^{3} \times 64$ lattice volume with an inverse lattice spacing of $1.73 \mathrm{GeV}$ and pion mass of $329 \mathrm{MeV}$. A complete calculation will be described which shows how the logarithmically divergent lattice contribution can be combined with continuum perturbation theory to obtain a physical result.

The 33rd International Symposium on Lattice Field Theory

14 - 18 July 2015

Kobe International Conference Center, Kobe, Japan*

\footnotetext{
* Speaker.

$\dagger$ This work was supported in part by U.S. DOE Grant No.DE-SC0011941 and used the BG/Q computing facilities of the RIKEN Brookhaven Research Center and the Brookhaven National Laboratory.
} 


\section{Indirect $\mathbf{C P}$ violation}

Indirect CP violation in the $K^{0}-\bar{K}^{0}$ system arises from mixing between the $\left|K^{0}\right\rangle$ and $\left|\bar{K}^{0}\right\rangle$ states and in the Standard Model is caused by $\Delta S=2$ weak interactions, second order in the Fermi constant $G_{F}$. The strength of this mixing is determined by the parameter $\varepsilon$ which has the experimental value $\varepsilon=2.228(11) \times 10^{-3}$. It is predicted by the Standard Model with less precision, $\varepsilon=2.13(23) \times 10^{-3}$ [四], where the dominant error comes from the uncertainly in the CKM matrix element $V_{c b}$. The two types of $W$-exchange diagrams which give rise to $\varepsilon$ in the standard model are shown in Fig. WThe largest contribution comes from short distances and the box diagram in Fig. ID dominates with the "disconnected" diagram in Fig. [b suppressed by $\alpha_{s}^{2}$. The short-distance contribution of the diagrams in Fig. W is described by the local, second order, effective weak Hamiltonian

$$
\mathscr{H}_{\mathrm{eff}}^{\Delta S=2}=\frac{G_{F}^{2}}{16 \pi^{2}}\left[\lambda_{c}^{2} \eta_{c c} S_{0}\left(x_{c}\right)+\lambda_{t}^{2} \eta_{t t} S_{0}\left(x_{t}\right)+2 \lambda_{c} \lambda_{t} \eta_{c t} S_{0}\left(x_{c}, x_{t}\right)\right] b(\mu) O_{L L}
$$

where $S_{0}$ is the Inami-Lim function, $\lambda_{q}=V_{q d}^{*} V_{q s}$ for $q=u, c$ and $t$ and $x_{q}=m_{q}^{2} / M_{W}^{2}$ with $M_{W}$ the $W$ boson mass. The correction factors $\eta_{c c}, \eta_{t t}$ and $\eta_{c t}$ provide higher order QCD corrections [[]] and factor $b(\mu)$ depends on the normalization scale $\mu$ used to define the operator $O_{L L}$ :

$$
O_{L L}=\bar{s} \gamma^{\mu}\left(1-\gamma^{5}\right) d \bar{s} \gamma^{\mu}\left(1-\gamma^{5}\right) d
$$

Lattice QCD is required to compute the matrix element of $O_{L L}$ between $\left|K^{0}\right\rangle$ and $\left|\bar{K}^{0}\right\rangle$ which is needed to determine $\varepsilon$, a matrix element usually expressed in terms of the parameter $B_{K}=$ $3\left\langle\bar{K}^{0}\left|O_{L L}\right| K^{0}\right\rangle / 8 f_{K}^{2} M_{K}^{2}$ which has been determined using lattice QCD to an accuracy of $2 \%$.

Given the current high-precision of $B_{K}$ and the prospects that $V_{c b}$ either becomes more accurately known or that $\varepsilon$ and the Standard Model might be used to determine it, we should examine the other errors affecting the calculation of $\varepsilon$. As emphasized by Buras, et al. [3] the box diagram in Fig. $\square$ also contributes to $\varepsilon$ when the two $W$ bosons are exchanged at positions that are separated by distances at the QCD scale. Such a "long-distance" contribution from each type of diagram shown in Fig. $\square$ can be represented by the diagrams shown in Fig. $\square$ where both $W$ propagators have been replaced by vertices and these vertices are integrated over QCD-scale separations.

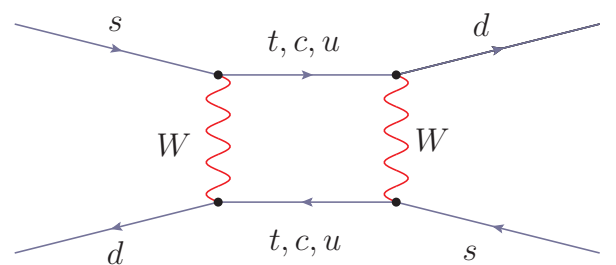

(a) Box

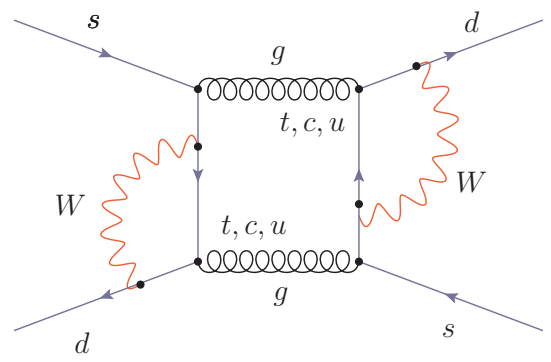

(b) Disconnected

Figure 1: The Inami-Lim box diagram (left) gives the largest contribution to $\varepsilon$. In that case one of the internal quarks is $t$ and the other $t$ or $c$. The right diagram shows the second possible topology which can contribute to $\varepsilon$ but which enters only at order $\alpha_{s}^{2}$. 
In order to understand such long-distance contributions, it is useful to recall more detail of the structure of the amplitudes described by the diagrams shown in Fig. W and the GIM cancellation that occurs. A convenient approach uses a Feynman path integral representation of the amplitudes contributing to $K^{0}-\bar{K}^{0}$ mixing and introduces a Feynman propagator $S_{q}[A](x, y)$ for a quark $q$ propagating in the background of a color gauge field $A_{\mu}(z)$, the path integral variable remaining after the fermion integrations have been performed. The sum over flavors for one of the two internal quark lines in both parts of Fig. $\square$ then can be written as follows:

$$
\begin{aligned}
\sum_{q=u, c, t} \lambda_{q} \gamma^{\mu}\left(1-\gamma^{5}\right) S_{q}[A] & (x, y) \gamma^{v}\left(1-\gamma^{5}\right) \\
& =\sum_{q=u, c, t} \lambda_{q} \gamma^{\mu}\left(1-\gamma^{5}\right)\left\{S_{q}[A](x, y)-S_{c}[A](x, y)\right\} \gamma^{v}\left(1-\gamma^{5}\right) .
\end{aligned}
$$

The left-hand side of Eq. ([1.3) shows the sum over the three types of "up" quark, weighted by the product of CKM matrix elements $\lambda_{q}=V_{q d}^{*} V_{q s}$. The right-hand side includes an Inami-Lim subtraction term which vanishes because of CKM unitarity: $\sum_{q=u, c, t} \lambda_{q}=0$. Equation (ㄸ.3) is nonstandard in that we subtract the charm- rather than the up-quark propagator which it ensures that $\mathrm{CP}$ violation comes only from one of the two non-zero terms in that equation.

This subtraction can be performed for each of the internal quark propagators in Fig. $\square$ and results in three terms for each, containing the products $\lambda_{t} \lambda_{t}, \lambda_{t} \lambda_{u}$, and $\lambda_{u} \lambda_{u}$. Only the first two products violate $\mathrm{CP}$ and only the $\lambda_{t} \lambda_{u}$ has significant long-distance contributions. The $u-c$ propagator difference in this $\lambda_{t} \lambda_{u}$ term realizes GIM cancellation. However, for the $t-c$ difference the $t$ and $c$ parts are best treated separately. The top-quark part is short-distance dominated and included in the usual expression in Eq. $(\mathbb{L} \mathbb{C})$. The charm-quark part is the subject of this talk.

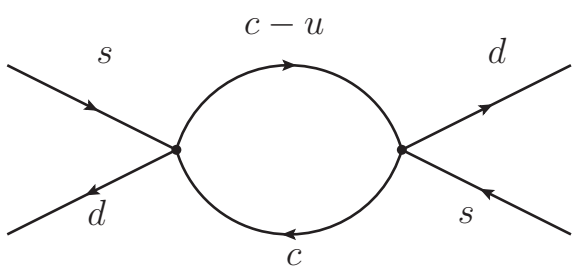

Figure 2: Diagram representing the longdistance contribution to $\varepsilon$ when the two $W$ propagators in Fig. 1a are reduced to points and allowed to separate on distances of the QCD scale.

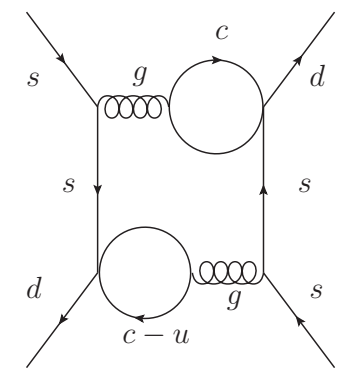

Figure 3: Diagram representing the longdistance contribution to $\varepsilon$ when the two $W$ propagators in Fig. $1 \mathrm{~b}$ are reduced to points.

The long-distance limit of the diagram in Fig. $\square$ can be easily described. The GIM cancellation leads to a logarithmically divergent loop integral whose divergence is regulated by the $W$ propagators present in its short distance structure and results in a $\ln \left(M_{W}^{2} / m_{c}^{2}\right) \approx 8$ term in the corresponding amplitude. This term is determined by the large momentum part of the integral and is included in

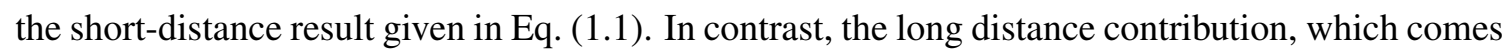
when the loop momentum is of order $m_{c}$ or smaller, is of order 1 .

The diagram shown in Fig. B is more complicated. Because of the GIM cancellation in the internal loop containing the charm and up propagator difference, this factor can be evaluated as 
shown. However, the second internal loop involving the charm quark alone will be logarithmically divergent and require the introduction of gluonic penguin operators to act as counter terms. Finally the entire graph is also logarithmically divergent, indicating a second $\ln \left(M_{W}^{2} / m_{c}^{2}\right)$ contribution.

\section{Second-order weak effective field theory}

In either a continuum or lattice calculation, the long distance contribution to $\varepsilon$ can be computed from a four-flavor low energy field theory containing $\Delta S=1$ and $\Delta S=2$ four-Fermi operators:

$$
H_{\mathrm{eff}}=H_{\mathrm{eff}}^{\Delta S=1}+C_{7} O_{L L}
$$

where the second order $\Delta S=2$ operator $O_{L L}$ is defined in Eq. (ㄷ.2) while $H_{\mathrm{eff}}^{\Delta S=1}$ is given by:

$$
H_{\mathrm{eff}}^{\Delta S=1}=\frac{G_{F}}{\sqrt{2}}\left\{\sum_{q, q^{\prime}=u, c} V_{q^{\prime} s}^{*} V_{q d} \sum_{i=1}^{2} C_{i} Q_{i}^{q^{\prime} q}+V_{t s}^{*} V_{t d} \sum_{i=3}^{6} C_{i} Q_{i}\right\} .
$$

Here the four-flavor, current-current operators are defined by:

$$
Q_{1}^{q^{\prime} q}=\left(\bar{s}_{i} q_{j}^{\prime}\right)_{V-A}\left(\bar{q}_{j} d_{i}\right)_{V-A} \quad Q_{2}^{q^{\prime} q}=\left(\bar{s}_{i} q_{i}^{\prime}\right)_{V-A}\left(\bar{q}_{j} d_{j}\right)_{V-A}
$$

with the repeated color indices $i, j$ summed over. The four QCD-penguin operators are given by

$$
\begin{aligned}
Q_{3} & =\left(\bar{s}_{i} d_{i}\right)_{V-A} \sum_{q=u, d, s, c}\left(\bar{q}_{j} q_{j}\right)_{V-A} & Q_{4} & =\left(\bar{s}_{i} d_{j}\right)_{V-A} \sum_{q=u, d, s, c}\left(\bar{q}_{j} q_{i}\right)_{V-A} \\
Q_{5} & =\left(\bar{s}_{i} d_{i}\right)_{V-A} \sum_{q=u, d, s, c}\left(\bar{q}_{j} q_{j}\right)_{V+A} & Q_{6} & =\left(\bar{s}_{i} d_{j}\right)_{V-A} \sum_{q=u, d, s, c}\left(\bar{q}_{j} q_{i}\right)_{V+A} .
\end{aligned}
$$

When an effective theory is used at second order, the first-order Hamiltonian must be augmented by new second-order terms whose coefficients are new "low energy constants" (LECs). These both remove new divergences which appear when loops are computed using the vertices of the first-order theory and provide information about new physics which is not visible in the firstorder theory. In our case $C_{7}$ is the single such second-order LEC, conventionally referred to as a Wilson coefficient. The $\overline{\mathrm{MS}}$ Wilson coefficients $C_{i}, 1 \leq i \leq 7$ are given in Ref. [D]]. In that calculation the $\Delta S=1$ coefficients $C_{i}, 1 \leq i \leq 6$ depend on the $\overline{\mathrm{MS}}$ renormalization scale $\mu$, compensating for the $\mu$ dependence of the renormalized operators $Q_{i}, 1 \leq i \leq 6$. However, the $\mu$ dependence of $C_{7}$ compensates not only for the $\mu$ dependence of the operator $Q_{7}$ but also for the $\mu$ dependence arising from the dimensionally regularized logarithmic divergence in the diagrams of Fig. .

\section{Lattice method}

We will now describe how the effective second-order theory specified by Eq. (2.]), viewed as a lattice regulated theory, can be used to calculate $\varepsilon$, including the long-distance contribution. Two types of $\left|\bar{K}^{0}\right\rangle-\left|K^{0}\right\rangle$ matrix elements are needed. The most familiar is that of the operator $O_{L L}$, used to calculate $B_{K}$. Much more challenging is the matrix element of the product of two $\Delta S=1$ operators $H_{\text {eff }}^{\Delta S=1}$, combined to give the second order amplitude:

$$
\mathscr{A}(T)=\frac{1}{2}\left\langle\bar{K}^{0}\left|\int_{0}^{T} d t_{1} \int_{0}^{T} d t_{2}\left\{H_{\mathrm{eff}}^{\Delta S=1}\left(t_{1}\right) H_{\mathrm{eff}}^{\Delta S=1}\left(t_{2}\right)\right\}\right| K^{0}\right\rangle .
$$


In Minkowski space this amplitude is equal to the integration time interval $T$ multiplied by the $K^{0}-\bar{K}^{0}$ mixing matrix $M_{\overline{0} 0}$, whose imaginary part determines $\varepsilon$. As is discussed in Ref. [四], the matrix element given in Eq. (B.D) can be also be obtained from the Euclidean-space lattice calculation of a four-point Greens function with initial and final interpolating fields for the $K^{0}$ and $\bar{K}^{0}$ and the two weak operators $H_{\text {eff }}^{\Delta S=1}\left(t_{1}\right)$ and $H_{\text {eff }}^{\Delta S=1}\left(t_{2}\right)$, integrated over time interval of length $T$, far-separated in time from the initial and final $K^{0}$ and $\bar{K}^{0}$ operators. In the Euclidean-space calculation, intermediate states with energy below $M_{K}$ give unphysical contributions with must be computed and removed. Finally, potentially important finite volume corrections must be made []].

The quantities $C_{7}\left\langle\bar{K}^{0}\left|O_{L L}\right| K^{0}\right\rangle$ and $\mathscr{A}(T)$ can then be combined to give $\varepsilon$ including both the short distance part from $O_{L L}$ and the long-distance part from $\mathscr{A}(T)$. However, before this can be done, we must determine the value for the Wilson coefficient $C_{7}$, appropriate for our lattice regularization. This is done by evaluating the complete, second-order Green's function in which incoming, external $\bar{s}$ and $d$ lines are connected to outgoing $s$ and $\bar{d}$ external lines. Following a generalized Rome-Southampton procedure, these external lines are evaluated off-shell at large non-exceptional momenta at a scale $\widetilde{\mu}$. The resulting amplitude is similar to that represented in Fig. $\square$ except that the simple tree graph with a four-quark vertex from $C_{7} O_{L L}$ must be added.

All of the quantities in this calculation are known except for $C_{7}$ which both a) absorbs the unphysical, high-momentum parts of the lattice-regulated loop integral shown in Fig. $\square$ and b) provides the short-distance, standard-model physics which contributes to $\varepsilon$. We can find $C_{7}$ by comparing the lattice result with the standard model result for this off-shell, gauge-fixed amplitude. For $\widetilde{\mu} \gg \Lambda_{\mathrm{QCD}}$ this off-shell Green's function can also be reliably computed from perturbation theory. The comparison of the lattice and perturbative results will then determine $C_{7}$ [ [⿴囗十, 目].

This procedure has been carried out as described in the next section. For this calculation, we evaluated the Inami-Lim box diagram in Fig. [a numerically at an external momentum scale $1.4 \leq$ $\tilde{\mu} \leq 2.6 \mathrm{GeV}$. While this may be an adequate first step, an important improvement is necessary. In computing the long-distance part of $\varepsilon$ we are extracting a term of order one in the presence of the larger short-distance part of order $\ln \left(M_{W}^{2} / m_{c}^{2}\right)$. Two-loop, $O\left(\alpha_{s}\right)$ corrections to this Inami-Lim function will then give $\alpha_{s} \ln \left(M_{W}^{2} / m_{c}^{2}\right)$ terms, similar in size to the $O(1)$ quantity we are computing. We are also neglecting the $O(1)$ leading- $\log \alpha_{s}^{n} \ln ^{n}\left(M_{W}^{2} / m_{c}^{2}\right)$ corrections that are typically included in a perturbative calculation of $C_{7}(\widetilde{\mu})$. These difficulties can be overcome if, instead of the InamiLim function, we begin with the effective theory given by Eq. (․․ determined perturbatively to all orders in $\alpha_{s}^{n} \ln ^{n}\left(M_{W}^{2} / m_{c}^{2}\right)$ [] ] and use it to calculate the off-shell Green's function to determine $C_{7}$. This matching calculation is carried out to one loop: the diagram of Fig. $\square$ is computed without additional QCD loops while the off-shell $C_{7} O_{L L}$ amplitude includes a one-loop, $O\left(\alpha_{s}\right)$ correction.

\section{Exploratory lattice calculation}

We use an ensemble with $N_{f}=2+1$ flavors of domain wall fermions, a $24^{3} \times 64$ lattice volume, an inverse lattice spacing of $1 / a=1.73 \mathrm{GeV}$ and pion, kaon and charm quark masses: $m_{\pi}=329 \mathrm{MeV}, m_{K}=5.75 \mathrm{MeV}$ and $m_{c}=941 \mathrm{MeV}$ respectively. In this first calculation we analyze only the diagrams labeled as type 1 and 2 in Ref. [四], omitting "double-penguin" (type 3) and disconnected (type 4) graphs as well as a fifth type of diagram with a new "single-penguin" topology. These added contractions have been evaluated but are not included here. 


\begin{tabular}{c|ccccc}
\hline$\widetilde{\mu}$ & 1.54 & 1.92 & 2.11 & 2.31 & 2.56 \\
\hline \hline $\operatorname{Im}\left(M_{0 \overline{0}}^{u t}\right)$ & $-5.642(64)$ & $-5.601(62)$ & $-5.586(61)$ & $-5.576(60)$ & $-5.511(58)$ \\
\hline
\end{tabular}

Table 1: Results for the imaginary part of the physical mixing matrix $M_{0 \overline{0}}^{u t}$ (in units of $10^{-15} \mathrm{MeV}$ ) for the $\lambda_{u} \lambda_{t}$ term as a function of the value the matching momentum scale $\widetilde{\mu}$ (in units of $\mathrm{GeV}$ ).

The calculation of $C_{7}$ appropriate to our lattice-regularized effective theory is done in two steps. First we evaluate the off-shell, Green's function containing four external quark lines and two factors of $H_{\text {eff }}^{\Delta S=1}$, integrated as in Eq. (B.D) and determine the $C_{7}(\widetilde{\mu})^{\mathrm{SD}-\text { lat }}$ needed to reproduce this result if instead the quantity $C_{7}(\widetilde{\mu})^{\mathrm{SD}-\text { lat }} O_{L L}$, integrated over the time interval $[0, T]$ replaces the bi-local operator in this off-shell Green's function. In the second step we use perturbation theory to determine the coefficient $C_{7}(\widetilde{\mu})^{\mathrm{SD}-\mathrm{PT}}$ chosen so that the off-shell Green's function containing $C_{7}(\widetilde{\mu})^{\mathrm{SD}-\mathrm{PT}} O_{L L}$ and evaluated at the scale $\widetilde{\mu}$ will reproduce the Inami-Lim function evaluated at the same external momenta. The combination $\left(C_{7}(\widetilde{\mu})^{\mathrm{SD}-\mathrm{PT}}-C_{7}(\widetilde{\mu})^{\mathrm{SD}-\mathrm{lat}}\right) O_{L L}$ can then be used in Eq. ([D) to define the effective lattice theory that is then evaluated numerically.

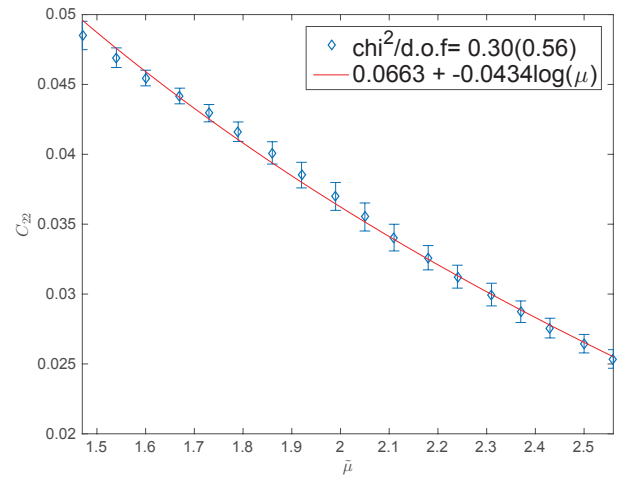

Figure 4: The $\widetilde{\mu}$ dependence of the $Q_{2} Q_{2}$ contribution to the off-shell Greens function used in determining $C_{7}^{\mathrm{SD}-\text { lat }}$.

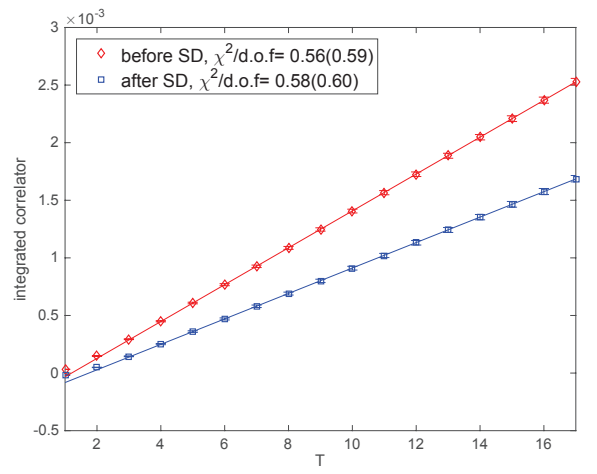

Figure 5: Contribution of $Q_{2} Q_{2}$ to $\mathscr{A}(T)$ with (lower) and without (upper) the short distance $C_{7}^{\text {SD-lat }}(\widetilde{\mu}=1.93 \mathrm{GeV})$ subtraction.

In Fig. $⿴$ we show the result for the coefficient $C_{7}^{\text {SD-lat }}$ determined from the contribution of the bi-local product of the operators $Q_{2} Q_{2}$ to the off-shell Green's function as a function of the offshell momentum scale $\widetilde{\mu}$. The result decreases with increasing $\widetilde{\mu}$ because the region of integration, lying between the scales $\widetilde{\mu}$ and $1 / a$, decreases as $\widetilde{\mu}$ increases. Figure $[$ shows the results for the product $Q_{2} Q_{2}$ with the short distance component, represented by $C_{7}(\widetilde{\mu})^{\mathrm{SD}-\text { lat }} O_{L L}$, either included or removed for the case $\widetilde{\mu}=1.92 \mathrm{GeV}$. The contribution to $\varepsilon$ is determined by the slope in $T$, which is reduced when the unphysical, lattice-regulated divergent part is removed.

Table $\square$ shows our preliminary results for $\operatorname{Im}\left(M_{0 \overline{0}}\right)$ as a function of the matching scale $\widetilde{\mu}$. While in an ideal calculation the $\widetilde{\mu}$ dependence should cancel between the lattice subtraction, $C_{7}(\widetilde{\mu})^{\mathrm{SD}-\text { lat }}$, the perturbative result for $C_{7}(\widetilde{\mu})^{\mathrm{SD}-\mathrm{PT}}$ and the scale dependence of $O_{L L}$, the very small $\widetilde{\mu}$ dependence seen in Table $\square$ should probably be viewed as fortuitous. 


\section{Conclusions}

In Eq. (5.J) we combine our result for the short- and long-distance contributions to the $\lambda_{t} \lambda_{u}$ part of $\varepsilon$ with the larger $\lambda_{t} \lambda_{t}$ part to give the following breakdown:

$$
\begin{gathered}
|\varepsilon|=(1.806(41)+0.891(11)+0.209(6)+0.112(13)) \times 10^{-3}=3.019(45) \times 10^{-3} \\
t t \quad u t_{S D} \quad u t_{L D} \quad \operatorname{Im}\left(A_{0}\right),
\end{gathered}
$$

where the lower line labels the source of each of the terms and only statistical errors are shown. The short- and long-distance parts of the $\lambda_{t} \lambda_{u}$ contribution correspond to the perturbative and the subtracted lattice contributions using the matching scale $\widetilde{\mu}=1.92 \mathrm{GeV}$. The final value in this equation is $50 \%$ larger than the $2.228(11) \times 10^{-3}$ experimental result because of our omission of the large logarithmic QCD corrections which arise when the Wilson coefficients at $\widetilde{\mu} \approx 2 \mathrm{GeV}$ are obtained from amplitudes at the $M_{W}$ scale. The difference between the 0.209 long-distance non-perturbative result in Eq. (5.J) and the 0.110 increase that results if the perburbative contribution is evaluated at $\widetilde{\mu}=0$ instead of $1.92 \mathrm{GeV}$, suggests $\approx 3 \%$ non-perturbative, long-distance contributions to $\varepsilon$.

Past experience in the calculation of the $K_{L}-K_{S}$ mass difference $\Delta M_{K}$ and the calculation presented here show that the long distance contribution to $\varepsilon$ is accessible to a first-principles, lattice QCD calculation. As is the case with $\Delta M_{K}$, we expect that the disconnected diagrams that have not been included in this exploratory calculation can be computed with current techniques and that the complete long-distance contribution can be computed with $\approx 10 \%$ statistical errors. The most significant challenge is working at a sequence of sufficiently small lattice spacings that an accurate continuum limit can be evaluated for these quantities which include a charm quark.

We thank C. Lehner for guidance using his PhySyHCAl framework [ []], and Christoph, X. Feng and our other RBC/UKQCD colleagues for many of the ideas on which this work is based.

\section{References}

[1] SWME Collaboration, J. A. Bailey, Y.-C. Jang, W. Lee and S. Park, Current status of $\varepsilon_{K}$ with lattice QCD inputs, arXiv:1503.06613 hep-lat].

[2] G. Buchalla, A. J. Buras and M. E. Lautenbacher, Weak decays beyond leading logarithms, Rev. Mod. Phys. 68 (1996) 1125-1144 [hep-ph/9512380].

[3] A. J. Buras, D. Guadagnoli and G. Isidori, On $\varepsilon_{K}$ beyond lowest order in the Operator Product Expansion, Phys.Lett. B688 (2010) 309-313 [arXiv:1002.3612 hep-ph]].

[4] RBC, UKQCD Collaboration, N. H. Christ, T. Izubuchi, C. T. Sachrajda, A. Soni and J. Yu, Long distance contribution to the KL-KS mass difference, Phys. Rev. D88 (2013) 014508 [arXiv:1212.5931 hep-1at].

[5] N. H. Christ, X. Feng, G. Martinelli and C. T. Sachrajda, Effects of finite volume on the KL-KS mass difference, Phys. Rev. D91 (2015), no. 11114510 [arXiv: 1504.01170 [hep-lat]].

[6] RBC, UKQCD Collaboration, N. Christ, T. Izubuchi, C. T. Sachrajda, A. Soni and J. Yu, Calculating the $K_{L}-K_{S}$ mass difference and $\varepsilon_{K}$ to sub-percent accuracy, PoS LATTICE2013 (2014) 397 [arXiv:1402.2577 hep-lat].

[7] C. Lehner, PhySyHCAl - A Physics System based on Hierarchical Computer Algebra, . http://physyhcal.lhnr.de. 